\title{
On turbulent friction in straight ducts with complex cross section: the wall law and the hydraulic diameter
}

\author{
SERGIO PIROZZOLI \\ Dipartimento di Ingegneria Meccanica e Aerospaziale, Sapienza Università di Roma \\ Via Eudossiana 18, 00184 Roma, Italy
}

\begin{abstract}
We develop predictive formulas for friction resistance in ducts with complex cross-sectional shape based on the use of the log law and neglect of wall shear stress nonuniformities. The traditional hydraulic diameter naturally emerges from the analysis as the controlling length scale for common duct shapes as triangles and regular polygons. The analysis also suggests that a new effective diameter should be used in more general cases, yielding corrections of a few percent to friction estimates based on the traditional hydraulic diameter. Fair but consistent predictive improvement is shown for duct geometries of practical relevance, including rectangular and annular ducts, and circular rod bundles.
\end{abstract}

\section{Introduction}

Internal flows within straight ducts having non-circular cross section are common in many applications of mechanical and hydraulic engineering, including water draining and ventilation systems, nuclear reactors, heat exchangers and turbomachinery. Despite their practical importance, flows in "complex" ducts are understood to a much lesser extent compared to canonical flows in plane channels and circular pipes, one of the main qualitative differences being the occurrence of secondary motions (Prandtl 1926; Nikuradse 1930). Of primary engineering importance is the prediction of the duct friction factor, namely

$$
\lambda=\frac{8 \bar{\tau}_{w}}{\rho u_{b}^{2}}
$$

where $\bar{\tau}_{w}$ is the average wall shear stress along the duct perimeter, $\rho$ is the fluid density, and $u_{b}$ is the duct bulk velocity. For that purpose, a widely used pragmatic approach is assuming that the same friction relationship can be used as for flow in circular pipes, provided a suitable Reynolds number is defined, based on a convenient duct length scale. The most traditional choice, apparently initiated by Schiller (1923), is the hydraulic diameter, defined as

$$
D_{h}=\frac{4 A}{P_{0}}
$$

where $A$ is the duct cross-sectional area, and $P_{0}$ is the duct perimeter. This choice is frequently justified by the appearance of the area/perimeter ratio in the mean momentum balance equations, which connects the mean wall friction to the duct pressure drop, namely

$$
\bar{\tau}_{w}=-\frac{A}{P} \frac{\mathrm{d} p}{\mathrm{~d} x}
$$




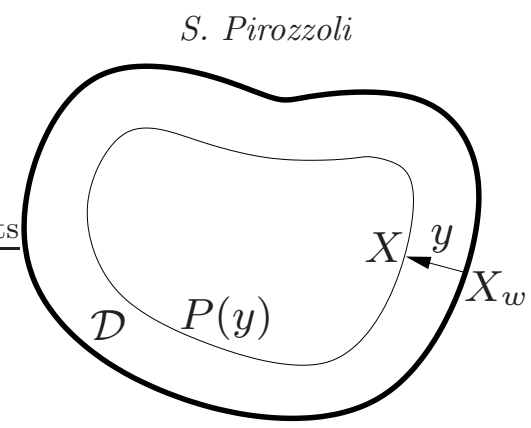

Figure 1: Typical duct cross section $(\mathcal{D})$, with indication of wall distance $y$, and associated area density, $P(y) . X$ is a generic point and $X_{w}$ is its wall foot.

but of course this is a quite tenuous argument. Indeed, limitations of the hydraulic diameter have emerged, especially in the study of ducts with aspect ratio (here broadly defined as the ratio of the largest to the smallest dimensions of the duct) significantly different than unity. Hence, semi-empirical corrections to the basic hydraulic diameter concept have been proposed over the years, tailored to specific duct geometries, as for instance rectangular ducts (Jones 1976), and general geometries have been frequently handled through ad-hoc extrapolation of laminar results (e.g. Maubach 1970; Rehme 1972). More elaborate techniques have sometimes been used relying on application of the log law in direction normal to the velocity iso-lines, which however require a cumbersome iterative procedure (Deissler \& Tavlor 1958).

One of the most robust findings in the (few) detailed quantitative studies of noncanonical duct flow is the presence of logarithmic layers in the wall-normal direction (e.g. Cain \& Duffy 1971; Nouri et al. 1993; Jonsson \& Sparrow 1966). Even more convincing support for the validity of the law-of-the-wall has come from recent numerical studies dealing with rectangular ducts (Vinuesa et al. 2014), square ducts (Pirozzoli et al. 2018), and hexagonal ducts (Marin et al. 2016), showing that in its inner form it applies with excellent approximation in the wall-normal direction up to the nearest corner bisector, even near the duct corners, provided the local wall friction is used. A formal theoretical analysis of turbulent flow in ducts with complex shape has been recently developed by Spalart et al. (2018). Extending classical inner-outer layer matching arguments, those authors deduced the validity of the logarithmic velocity profile, but also arrived at the interesting prediction that the wall friction should tend towards being uniform all around the duct except near possible corners, asymptotically as $R e \rightarrow \infty$. This was confirmed by numerical solutions obtained with turbulence models, and it is consistent with recent DNS at moderately high Reynolds number (Pirozzoli et al. 2018). Hence, a reasonably simple structure of turbulent flow in ducts emerges, which lends itself to analytical treatment. In the forthcoming $\$ 2$ we develop a simple predictive formula for the friction coefficient for ducts with arbitrary shape, and in $\S 3$ we test its predictive capability. Last, in $₫ 4$ we outline implications of the present findings.

\section{Friction estimates}

The forthcoming discussion pertains to straight ducts with cross-sectional shape $\mathcal{D}$ such that a wall distance $y$ (defined at the normal distance to the closest wall) can be defined for any point $X$, as depicted in figure1 This is a generally acceptable assumption, and counterexamples are difficult to conceive. We hereafter further assume that: 
(a) the inner-layer law-of-the wall applies with respect to the closest wall, hence

$$
\bar{u}(X) / u_{\tau}=f\left(y u_{\tau} / \nu\right),
$$

where $u_{\tau}=\left(\tau_{w} / \rho\right)^{1 / 2}$ is the local friction velocity at the wall point $X_{w}$, and $f$ is a suitable functional inner form for the wall law;

(b) the wall shear stress is constant along the duct perimeter, hence $\tau_{w}=\bar{\tau}_{w}$.

Assumption (a) is supported by all available experimental and numerical data (Cain \& Duffy 1971; Jonsson \& Sparrow 1966; Nouri et al. 1993; Marin et al. 2016; Nikitin 2006). For instance, in square ducts (Pirozzoli et al. 2018) clear logarithmic layers are found to form at bulk Reynolds number barely exceeding $10^{4}$. Small flow-dependent deviations from the strict inner law are present in the core part of the flow, as for instance in circular pipes and plane channels, which will be neglected here for convenience. Assumption (b) is probably weaker, as direct detailed measurements of the local wall friction distribution are scarce, and numerical simulations are limited to relatively low Reynolds numbers. The available data show that, with the obvious exception of sharp corners where the friction is zero, variations of the wall shear stress in simply-connected ducts are no more that 10\% (Leutheusser 1963; Cain \& Duffy 1971; Marin et al. 2016). In the best documented case of square ducts, variations of local wall friction are further found to very nearly cancel out as far as their contribution to the mean friction coefficient is concerned (Pirozzoli et al. 2018). Compelling theoretical arguments (Spalart et al. 2018) do in fact suggest that flat distributions of the wall shear stress should result in the asymptotic high-Reynolds-number limit. Larger variations of wall friction have been reported in multiply-connected ducts, as discussed in $\$ 3.2$ Although certainly criticizable, the assumptions (ㅁa),(b) are the only viable pathway to arrive at closed form predictions, hence their validity may be judged from the outcome. Similar assumptions were also used by Keulegan (1938) to estimate friction in open channels with trapezoidal cross section, and are frequently used in the hydraulics community. However, it appears that implications have not been fully pursued in the past, especially within the context of internal flows.

A direct consequence of the above assumptions is that the mean streamwise velocity only depends on the inner-scaled wall distance according to

$$
u^{*}=f\left(y^{*}\right),
$$

where the star denotes normalization with global wall units, namely $u^{*}=\bar{u} / u_{\tau}^{*}, y^{*}=$ $y u_{\tau}^{*} / \nu$, with $u_{\tau}^{*}=\left(\bar{\tau}_{w} / \rho\right)^{1 / 2}$. The bulk velocity in the duct is then easily deduced by integration, yielding

$$
u_{b}^{*}=\frac{1}{A} \int_{\mathcal{D}} f\left(y^{*}\right) \mathrm{d} A .
$$

The integration is conveniently carried out by introducing the area density with respect to the wall distance, $P(y)=\mathrm{d} A / \mathrm{d} y$ (see figure 1), which may be interpreted as the perimeter associated with the iso- $y$ lines, with $P(0)=P_{0}$, thus obtaining

$$
u_{b}^{*}=\frac{1}{A} \int_{0}^{y_{m}} P(y) f\left(y^{*}\right) \mathrm{d} y=\int_{0}^{1} \tilde{P}(\eta) f\left(\eta \cdot y_{m}^{*}\right) \mathrm{d} \eta,
$$

where $y_{m}$ is the maximum wall distance over the duct cross section, $\eta=y / y_{m}$ is the normalized wall distance, $y_{m}^{*}=y_{m} u_{\tau}^{*} / \nu$ is the counterpart of the friction Reynolds number in canonical flows, and $\tilde{P}(\eta)=P\left(\eta y_{m}\right) / \bar{P}$ is the normalized perimeter function, with $\bar{P}=A / y_{m}$ the mean duct perimeter. Equation (2.3) makes it clear that the wall-scaled bulk velocity, and hence the friction factor (from equation (1.1), $\lambda=8 / u_{b}^{* 2}$ ), only depends upon the wall function, which is here assumed to be the same for all ducts, and 
on the duct geometry through the inner-scaled maximum distance $y_{m}^{*}$, and the normalized perimeter function. For instance, in the case of a circular pipe with diameter $D$ it is straightforward to verify that $y_{m}=D / 2, \bar{P}=\pi D / 2$, and $\tilde{P}=2(1-\eta)$. Notably, very similar conclusions are obtained for any triangle and for regular polygons, in which case $y_{m}$ is the apothem of the cross section, $\bar{P}$ is half of the outer perimeter, and again $\tilde{P}=2(1-\eta)$. This observation leads to the first important conclusion that, based on the previous assumptions, triangles and regular polygons should share the same friction relation, provided the apothem is used as length scale in the definition of the Reynolds number. However, it is easy to show that for all these geometries the hydraulic diameter defined in equation (1.2) is two times the duct apothem, hence we find that the assumed strict validity of the wall law yields as a direct, exact consequence, that the hydraulic diameter is the proper length scale to achieve universality of the friction coefficient distribution.

Additional elaborations can be made by assuming a logarithmic form for the law-ofthe-wall which formally encompasses both the case of smooth and rough walls, namely

$$
u^{*}=\frac{1}{k} \log \left(y^{*} / y_{0}^{*}\right)=\frac{1}{k} \log \left(y / y_{0}\right),
$$

where $k$ is the von Karman constant, and $y_{0}$ is the virtual origin for the wall law, defined as (Colebrook 1939)

$$
y_{0}=\frac{\alpha \nu}{u_{\tau}^{*}}+\beta \varepsilon,
$$

where $\varepsilon$ is the equivalent sand-grain roughness height, and $\alpha \approx 1 / 10, \beta \approx 1 / 33$. Partial integration of (2.3) yields

$$
u_{b}^{*}=\frac{1}{k} \log \left(y_{m} / y_{0}\right)+\frac{1}{k} \underbrace{\int_{0}^{1} \tilde{P}(\eta) \log \eta \mathrm{d} \eta}_{\mathcal{C}},
$$

where $\mathcal{C}$ is solely a function of the duct geometry. The same asymptotic Reynolds number trend also directly stems from matching arguments (Spalart et al. 2018), however the analysis is here completed by integration over the cross section, thus yielding full friction predictions.

In the case of flow in a circular pipe, Eqn. (2.6) becomes

$$
u_{b}^{*}=\frac{1}{k} \log \left(D / 2 / y_{0}\right)-\frac{3}{2 k} .
$$

Comparing equations (2.6) and (2.7) shows that the two formulas are identical provided $D$ in equation (2.7) is replaced with

$$
D_{e}=2 y_{m} e^{3 / 2+\mathcal{C}},
$$

which may then be defined as an effective diameter for the duct, namely the diameter of a circular pipe yielding the same friction coefficient. As previously pointed out, the effective diameter herein predicted coincides with the traditional hydraulic diameter in ducts with triangular and regular polygonal shape, for which $\mathcal{C}=-3 / 2$. Equation (2.8) however highlights that the correct length scale to achieve universality of the friction law is in general different than the hydraulic diameter. Differences in the prediction of $C_{f}$ may be estimated considering the extreme case of an infinitely wide channel with height $H$, whose hydraulic diameter is $D_{h}=2 H$, and whose normalized perimeter function is $\tilde{P} \equiv 1$. From equation (2.8) it follows that $D_{e}=\sqrt{e} H \approx 1.65 H$, which by construction returns the log-law based friction law for plane channel flow. Assuming for simplicity 
(a)

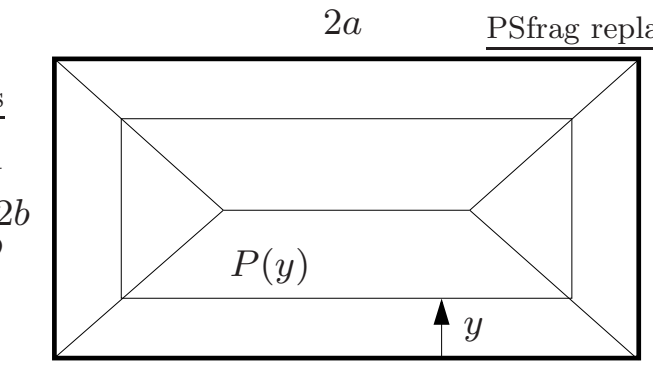

(b)

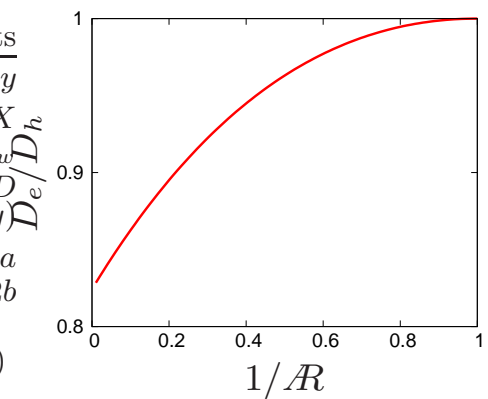

Figure 2: Rectangular ducts: (a) cross section with indication of wall distance $y$, and associated area density, $P(y)$; (b) correction factor as a function of duct inverse aspect ratio.

$\lambda \sim R e_{D}^{-1 / 4}$ Blasius 1913), we estimate that use of the hydraulic diameter in this case yields underestimation of the friction coefficient of about $5 \%$, as indeed confirmed by the later results.

In the following we verify the predictive power of the traditional hydraulic diameter and of the effective diameter (2.8) for smooth ducts with relatively complex geometry, and for which a sufficient number of experimental measurements are available.

\section{Applications}

\subsection{Rectangular ducts}

Rectangular ducts have been extensively studied in the literature because of their wide range of use. Limited success of the classical hydraulic diameter concept is recognized in this case, and corrections have been proposed, the most frequently used (Jones 1976) being based on the definition of a laminar-equivalent hydraulic diameter such that the friction coefficient in the laminar regime is the same for ducts with any aspect ratio. For a rectangular duct with sides length $a \geqslant b$, and aspect ratio $R=a / b$ (see figure 2), the hydraulic diameter is $D_{h}=4 a b /(a+b)$, the maximum wall distance is $y_{m}=b$, the normalized perimeter function defined in equation (2.3) is given by

$$
\tilde{P}=\frac{1+R-2 \eta}{R},
$$

and the geometric factor $\mathcal{C}$ defined in equation (2.6) is

$$
\mathcal{C}=-1-\frac{1}{2 \pi}
$$

It follows that the effective diameter is

$$
D_{e}=2 b e^{\frac{\mathscr{R}-1}{2 A R}}=\frac{1+R}{2 R} D_{h} e^{\frac{\not R-1}{2 A R}},
$$

as graphically shown in figure 2. It should be noted that equation (3.3) predicts that the hydraulic diameter is the correct length scale for square ducts, in line with DNS data at $R e_{\tau} \approx 1000$ (Pirozzoli et al. 2018).

Figure 3 shows the measured friction coefficient in rectangular ducts with aspect ratios up to 31, as collected in the work of Jones (1976), as a function of the traditional hydraulic diameter (left panel) and as a function of the effective hydraulic diameter defined in equation (3.3) (right panel). For reference, the Kármán-Prandtl friction law for smooth 
(a)

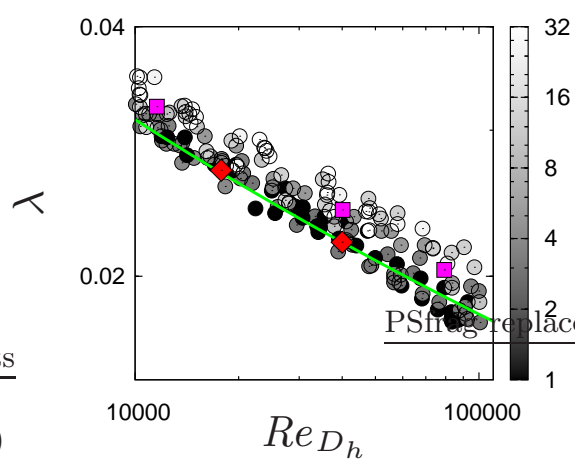

(b)

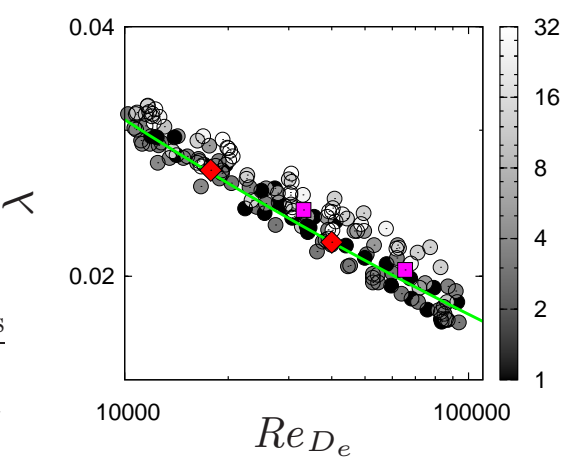

Figure 3: Experimentally measured friction factor for rectangular ducts with various aspect ratios (Jones 1976), as from color scale. Solid symbols denote DNS data for square duct (Pirozzoli et al. 2018, diamonds), and plane channel flow (Bernardini et al. 2014, squares). Left, expressed as a function of the hydraulic diameter; right, expressed as a function of the effective diameter, equation 3.3 The solid line denotes the KármánPrandtl friction law for a smooth circular pipe. The relative standard error is $8.1 \%$ in panel (a), and $5.7 \%$ in panel (b).

circular pipes

$$
1 / \lambda^{1 / 2}=2 \log _{10}\left(\operatorname{Re}_{D} \lambda^{1 / 2}\right)-0.8,
$$

is also shown, as well as DNS data for square ducts and plane channels. As expected based on the previous discussion, use of the hydraulic diameter yields systematic underprediction of the friction factor for ducts with high aspect ratio. This is particularly true for a subset of experimental data which even overshoot friction of plane channel flow, thus raising doubts about their reliability. Despite significant scatter among experimental data, it appears that the log-law based effective diameter yields better universality of the distributions, especially insofar as it tends to shift data points corresponding to highaspect-ratio ducts closer to the circular pipe case, including the infinite aspect ratio case. The remaining differences between plane channel and pipe flow, of about $2 \%$, are likely due to differences in the core velocity profiles, here neglected. In fact, the wake region in plane channel flow is weaker than in pipes, hence the friction coefficient is higher, all the rest being the same. Similar results as shown in figure 3(b) may be obtained from semi-empirical corrections (see Jones 1976), which however have very tenuous theoretical foundation.

\subsection{Annular ducts}

Flows in annular passages are common in mechanical engineering, and for instance they are important in drilling wells, where mud passes through the drill shaft and the well casing to remove cuttings and friction-generated heat. Let $L$ be the offset between the inner cylinder with radius $R_{1}$, and the outer cylinder with radius $R_{2}$, the geometry (see figure $4(\mathrm{a})$ ) is controlled by two parameters, namely the diameter ratio $\delta=R_{2} / R_{1}$, and the eccentricity, $\epsilon=L /\left(R_{2}-R_{1}\right)$. The hydraulic diameter is simply $D_{h}=2\left(R_{2}-R_{1}\right)$, bearing no dependence on $\delta$ and $\epsilon$. It is a simple matter to show that the maximum wall distance is in this case $y_{m}=1 / 2\left(R_{2}-R_{1}\right)(1+\epsilon)$, and the mean perimeter is $\bar{P}=A / y_{m}=2 \pi\left(R_{2}+R_{1}\right) /(1+\epsilon)$. After some algebra, the following expression is 
(a)

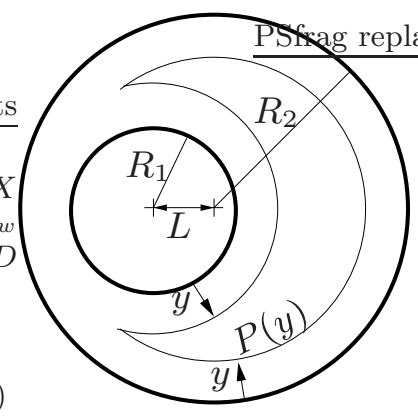

(b)

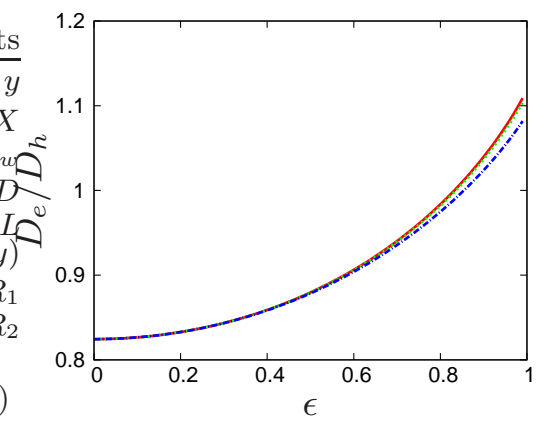

Figure 4: Annular ducts: (a) cross section with indication of wall distance $y$, and associated area density, $P(y)$, with $R_{1}$ and $R_{2}$ the radii of the inner and outer cylinder, respectively, and $L$ the offset between their centers; (b) correction factor as a function of the duct eccentricity $\epsilon$ for various duct diameters ratio: $\delta=1.33$ (solid), $\delta=1.78$ (dashed), $\delta=3.56$ (dot-dashed).

(a)

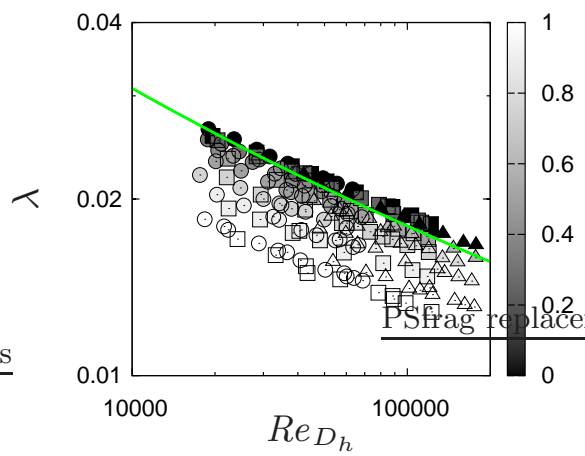

(b)

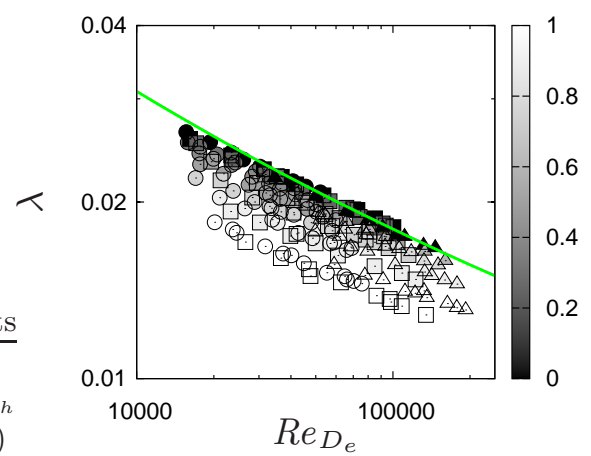

Figure 5: Experimentally measured friction factor for annular ducts with different eccentricities (Jonsson \& Sparrow 1966), as from color scale, and different diameters ratio: $\delta=1.33$ (circles); $\delta=1.78$ (squares), $\delta=3.56$ (triangles). Left, expressed as a function of the hydraulic diameter; right, expressed as a function of the effective diameter, equation 2.8 The solid line denotes the Kármán-Prandtl friction law for a smooth circular pipe. The relative standard error for $\epsilon \leqslant 0.25$ is $3.0 \%$ in panel (a), and $2.5 \%$ in panel (b).

obtained for the normalized perimeter function

$$
\tilde{P}(\eta)= \begin{cases}1+\epsilon, & \text { for } 0 \leqslant \eta \leqslant \eta^{*} \\ \frac{1+\epsilon}{\pi(\delta+1)}\left[\delta \theta_{2}+\theta_{1}+\eta / 2(\delta-1)(1+\epsilon)\left(\theta_{1}-\theta_{2}\right)\right], & \text { for } \eta^{*} \leqslant \eta \leqslant 1\end{cases}
$$

where $\eta^{*}=(1-\epsilon) /(1+\epsilon)$,

$\cos \theta_{1}=\frac{(\delta+1)[(1+\epsilon) \eta-1]+\epsilon^{2}(\delta-1)}{\epsilon(\delta-1)[2+\eta(\delta-1)(1+\epsilon)]}, \quad \cos \theta_{2}=\frac{(\delta+1)[(1+\epsilon) \eta-1]-\epsilon^{2}(\delta-1)}{\epsilon(\delta-1)[2 \delta-\eta(\delta-1)(1+\epsilon)]}$.

Numerical integration of function $\mathcal{C}$ defined in equation (2.6), with $\tilde{P}$ given in equation (3.5) yields the result shown in figure $4(\mathrm{~b})$. As in periodic channel flow, the corrective factor over the hydraulic diameter is $\sqrt{e} / 2$ at zero eccentricity, becoming closer and exceeding unity at increasing values of $\epsilon$. The dependence on the diameter ratio is quite weak, and confined to high values of $\epsilon$, a good fit for the data at $\epsilon \lesssim 0.6$ being $D_{e} / D_{h}=\sqrt{e} / 2+0.217 \epsilon^{2}$. 
8

(a)

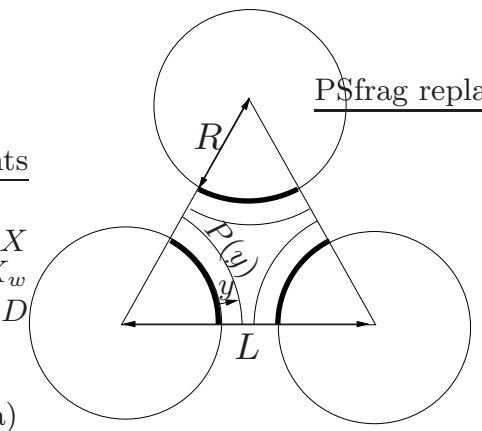

(b)

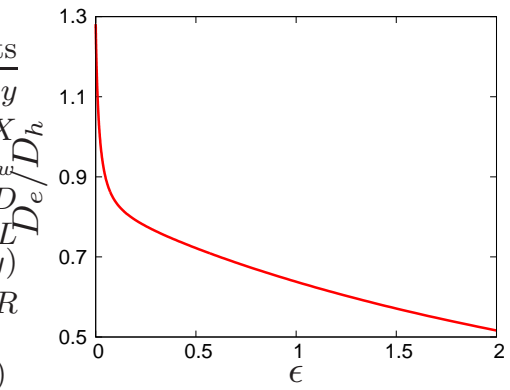

Figure 6: Rod bundles in hexagonal arrangement: (a) elementary module with indication of wall distance $y$, and associated area density, $P(y)$, with $R=D / 2$ the rod radius, and $L$ the pitch between neighboring rods centers; (b) correction factor as a function of pitch parameter $\epsilon=L / D-1$.

Extensive measurements of concentric and eccentric pipe flows were carried out by Jonsson \& Sparrow (1966), in a wide range of duct eccentricities and diameters ratio. Those authors found that the wall shear stress varies in the circumferential direction proportionally to the duct eccentricity, with larger shear at the location of the widest gap. Further difficulties are associated with the occurrence of locally laminar flow at the smallest gap (Nikitin 2006). Comparison of the classical hydraulic diameter representation with predictions of equation (2.8) are shown in figure 5. The classical hydraulic diameter scaling (left panel) shows higher friction than given by the Kármán-Prandtl friction law at low duct eccentricity, and substantially lower at high eccentricity, mostly associated with the formation of regions of laminar flow. The effective diameter is partially successful in shifting the friction data for ducts with low eccentricity $(\epsilon \lesssim 0.25$, see color scale and figure caption) towards the universal distribution. Although pointing in the right direction (recalling figure $4(\mathrm{~b})$ ) the effective diameter doesn't yield the same satisfactory behavior also at higher eccentricity, which is probably not unexpected as the underlying assumption of uniform wall stress is severely invalidated.

\subsection{Circular rod bundles}

The prediction of friction in bundles of rods with circular cross section is important in the cooling channels of nuclear reactors, hence this flow has been extensively characterized in the past. Although several arrangements of rods are possible, here we consider an hexagonal arrangement, under the assumption of nominally infinite number of rods, so as to neglect boundary effects related to the presence of confining walls. The relevant geometrical molecule for the study of this flow is shown in figure 6(a). The geometry of the typical cross section is identified through a single parameter, namely the pitch/diameter ratio, $L / D=1+\epsilon, \epsilon=0$ corresponding to the limit case of tangent rods. The hydraulic diameter for the typical cross section is (Rehme 1973)

$$
D_{h}=D(1+\epsilon)^{2} \frac{\tan \alpha}{\alpha},
$$

with $\alpha=\pi / 6$. It turns out that the maximum wall distance is $y_{m}=R((1+\epsilon) \cos \alpha-1)$, the average perimeter is

$$
\bar{P}=\frac{3\left[(1+\epsilon)^{2} \sin \alpha-\alpha \cos \alpha\right]}{(1+\epsilon)-\cos \alpha},
$$


(a)

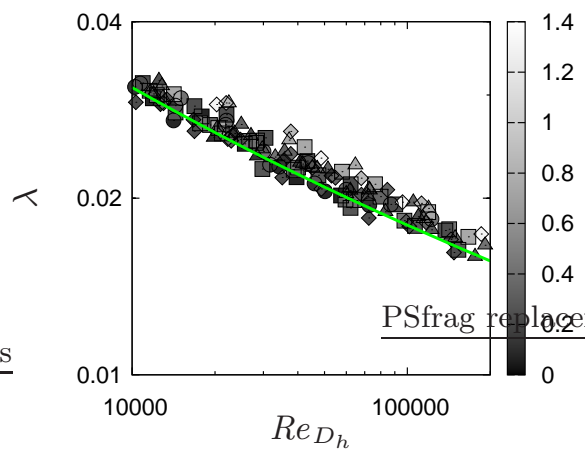

(b)

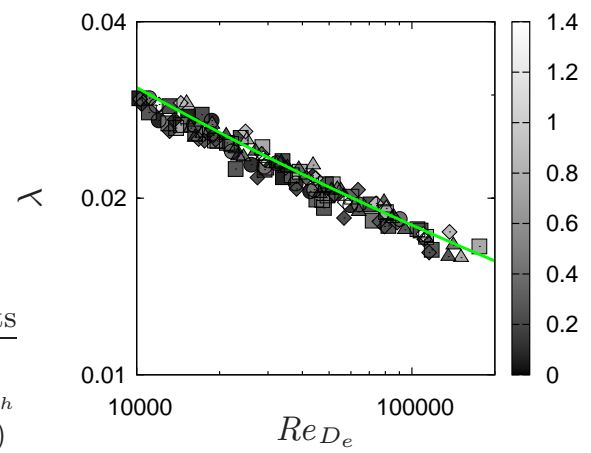

Figure 7: Experimentally measured friction factor for rod bundles for different pitch parameters $\epsilon$ Rehme 1973), as from color scale, and different numbers of rods: $N=61$ (circles); $N=37$ (squares), $N=19$ (triangles), $N=7$ (diamonds). Left, expressed as a function of the hydraulic diameter; right, expressed as a function of the effective diameter, equation 2.8. The solid line denotes the Kármán-Prandtl friction law for a smooth circular pipe. The relative standard error is $6.8 \%$ in panel (a), and $4.5 \%$ in panel (b).

and the normalized perimeter function is

$$
\tilde{P}(\eta)=\frac{6 R}{\bar{P}}\left\{\begin{array}{ll}
\alpha\left(1+\eta y_{m} / R\right), & \text { for } 0 \leqslant \eta \leqslant \eta^{*} \\
\left(1+\eta y_{m} / R\right)\left[\alpha-\arccos \left(\frac{1+\epsilon}{1+\eta y_{m} / R}\right)\right], & \text { for } \eta^{*} \leqslant \eta \leqslant 1
\end{array},\right.
$$

where $\eta^{*}=\epsilon R / y_{m}$.

Numerical integration of equation (2.6) yields the effective diameter as a function of the parameter $\epsilon$, as shown in figure 6(b). The effective diameter is found to be greater than the hydraulic diameter for small $\epsilon$, with $D_{e} / D_{h} \rightarrow 1.281$ as $\epsilon \rightarrow 0$, and decreasing as $1 / \epsilon$ for large $\epsilon$.

In figure 7 we show experimental data by Rehme (1973) in the range of pitch parameters $0.025 \leqslant \epsilon \leqslant 1.320$. When normalized with respect to the hydraulic diameter (left panel), the data show consistently higher friction than expected, the higher is $\epsilon$. The effective diameter (right panel) does eliminate most large overshoots, reestablishing the universal friction law for all data at sufficiently high pitch ratio (light shades). Data points corresponding to small values of $\epsilon$ are overestimated, presumably because flow becomes locally laminar in the presence of narrow gaps, an effect which again cannot be captured given the initial assumptions.

\section{Conclusions}

We have derived predictive formulas for friction in ducts with complex shape, under the assumption that the friction distribution along the duct perimeter is uniform, and that the velocity at any given point in the cross section is controlled by the nearest wall through an assumed inner-layer log law, upon neglect of core deviations. The leading conclusion is that the classical hydraulic diameter is the proper length for many common duct shapes such as triangles and regular polygons, thus providing theoretical support to its widespread use. This finding is supported by a number of recent DNS in polygonal ducts which with very good precision show collapse of friction data on the universal Kármán-Prandtl distribution. A second important conclusion is that deviations from the classical hydraulic diameter scaling should arise in more general duct shapes, for 
which the effective diameter defined in equation (2.8) is expected to be a more accurate choice. The latter can be easily evaluated, either analytically or numerically, based on the duct cross-sectional shape. Differences with respect to friction predictions based on the classical hydraulic diameter are generally small, but more sensible as the duct aspect ratio is much different than unity, amounting in practical terms to a few percent. Reevaluation of classical experiments in smooth ducts with moderately complex shape, namely rectangular and annular ducts, and circular rod bundles, supports small but consistent predictive improvements when the effective diameter is used instead of the traditional hydraulic diameter, with all due caveats incurred with tracing differences of a few percent within scattered experimental data. Of course, given the assumptions made in the derivation, the log-law based effective diameter does not perform well in cases in which the wall shear stress is far from uniform, such as ducts with narrow gaps or acute angles, which may even feature locally laminar flow. Measurements at higher Reynolds number and/or for rough ducts would be desirable, to be able to more clearly ascertain

the predictive power of the log-law based effective diameter, and to compare with existing correlations based on extrapolation of laminar results.

This paper has benefited from exchange of ideas with P. Orlandi and P.R. Spalart.

\section{REFERENCES}

Bernardini, M., Pirozzoli, S. \& Orlandi, P. 2014 Velocity statistics in turbulent channel flow up to $R e_{\tau}=4000$. J. Fluid Mech. 742, 171-191.

Blasıus, H. 1913 Das ähnlichkeitsgesetz bei reibungsvorgängen in flüssigkeiten. In Mitteilungen über Forschungsarbeiten auf dem Gebiete des Ingenieurwesens (ed. Verein deutscher Ingenieure), , vol. 131, pp. 1-41. Springer-Verlag.

Cain, D. \& Duffy, J. 1971 An experimental investigation of turbulent flow in elliptical ducts. Int. J. Mech. Sci. 13, 451-459.

Colebrook, C.F. 1939 Turbulent flow in pipes, with particular reference to the transition region between the smooth and rough pipe laws. J. Inst. Civil Eng. 11, 133-156.

Deissler, R.G. \& TAYlor, M.F. 1958 Analysis of turbulent flow and heat transfer in noncircular passages. Tech. Rep. TN-4384. NACA.

Jones, O.C. 1976 An improvement in the calculation of turbulent friction in rectangular ducts. ASME J. Fluids Engng. 98, 173-181.

Jonsson, V.K. \& Sparrow, E.M. 1966 Experiments on turbulent-flow phenomena in eccentric annular ducts. J. Fluid Mech. 25, 65-86.

Keulegan, G.H. 1938 Laws of turbulent flow in open channels. J. Res. Nat. Bureau Stand. 21, 707-741.

Leutheusser, H.J. 1963 Turbulent flow in rectangular ducts. J. Hydr. Div. ASCE 89 (3), 1-19.

Marin, O., Vinuesa, R., Obabko, A.V. \& Schlatter, P. 2016 Characterization of the secondary flow in hexagonal ducts. Phys. Fluids 28 (12), 125101.

Maubach, K. 1970 Reibungsgesetze turbulenter strömungen. Chemie Ingenieur Technik 42 (15), 995-1004.

Nikitin, N. 2006 Direct numerical simulation of turbulent flows in eccentric pipes. Comp. Math. Math. Phys. 46, 489-504.

Nikuradse, J. 1930 Turbulente strömung in nicht-kreisförmigen rohren. Ing. Arch. 1, 306-332.

Nouri, J.M., Umur, H. \& Whitelaw, J.H. 1993 Flow of newtonian and non-newtonian fluids in concentric and eccentric annuli. J. Fluid Mech. 253, 617-641.

Pirozzoli, S., Modesti, D., Orlandi, P. \& Grasso, F. 2018 Turbulence and secondary motions in square duct flow. J. Fluid Mech. 840, 631-655.

Prandtl, L. 1926 Über die ausgebildete turbulenz. In Int. Congress for Applied Mechanics. Also "Turbulent Flow", NACA-TM 435, 1927.

Renme, K. 1972 Pressure drop performance of rod bundles in hexagonal arrangements. Int. J. Heat Mass Transf. 15 (12), 2499-2517. 
Rehme, K. 1973 Simple method of predicting friction factors of turbulent flow in non-circular channels. Int. J. Heat Mass Transf. 16 (5), 933-950.

SCHILlER, L. 1923 Über den strömungswiderstand von rohren verschiedenen querschnitts und rauhigkeitsgrades. ZAMM-Zeitschrift für Angewandte Mathematik und Mechanik 3 (1), 213.

Spalart, P.R., Garbaruk, A. \& Stabnikov, A. 2018 On the skin friction due to turbulence in ducts of various shapes. J. Fluid Mech. 838, 369-378.

Vinuesa, R., Noorani, A., Lozano-Durán, A., Khoury, G.K.E., Schlatter, P., Fischer, P.F. \& NAGIB, H.M. 2014 Aspect ratio effects in turbulent duct flows studied through direct numerical simulation. J. Turbulence 15 (10), 677-706. 